\title{
THE GROUP OF SELF-HOMOTOPY EQUIVALENCES OF $S^{2}$-BUNDLES OVER $S^{4}$, I
}

\author{
Dedicated to Professor Nobuo Shimada on his 60th birthday
}

BY KOHHEI YAMAGUCHI

\section{Introduction.}

The set $E q(X)$ of homotopy classes of self-homotopy equivalences of a based space $X$ forms a group under the composition of maps, and it is called the group of self-homotopy equivalences of $X$. The group $E q(X)$ has been studied by several authors since the paper of W. D. Barcus and M. G. Barratt [1] appeared.

However, we have not yet obtained an effective method for calculating it except classical ones, and its structure also has not been clarified sufficiently. Furthermore, very little is known about this group even when $X$ is a simply connected $C W$ complex with three cells which is not a $H$-space. In particular, when $X$ is a total space of $S^{m}$-bundles over $S^{n}$, the group $E q(X)$ was already considred for $X=S^{m} \times S^{n}$ in [7], [8], [17], for a principal $S^{3}$-bundle over $S^{n}$ in [9], [13], [16], and for the real and complex Stiefel manifolds $W_{n, 2}$ and $V_{n, 2}$ in [10]. Recently, S. Sasao studied the group $E q(X)$ in [15] for the total space of $S^{m}$-bundles over $S^{n}$ under the stable range, $3<m+1<n<2 m-2$.

On the othe hand, it seems to be very difficult to investigate it under the unstable range. However, we would like to consider it when $X$ is simply connected and the total space of $S^{m}$-bundles over $S^{n}$ for a small pair of integers $(m, n)$. Since $X$ is simply connected, $n, m \geqq 2$ and the cases $(m, n)=(2,2)$ or $(2,3)$ were already considered by P. J. Kahn [7] and N. Sawashita [8].

Then the purpose of this paper is to study the group $E q(X)$ for the case $(m, n)=(2,4)$ and we will treat its application in the subsequent paper in [22].

\section{Notations and Results.}

All spaces have base points, and all maps and homotopies preserve base points throughout this note. We denote by $[X, Y]$ the set of based homotopy classes of maps from $X$ to $Y$, and we will not distinguish between a map and its homotopy class. Let $Z\{x\}$ (resp. $Z_{m}\{x\}$ ) be the infinite cyclic group (resp. the cyclic group of order $m$ ) generated by the element $x$. Let $R P^{n}$ (resp. $C P^{n}$ )

Received January 28, 1986 
and $S O_{n}$ be the $n$-dimensional real (resp. complex) projective space and the $n$-th rotation group, respectively. We denote by the map $\rho: S^{3} \rightarrow R P^{3}=S_{3}$ the double covering projection, and it is trivial to see $\pi_{3}\left(S_{3}\right)=Z\{\rho\}$. In the exact sequence $A \rightarrow B \rightarrow C \rightarrow 1$, we write the group composition in the group $A$ as addition, and the compositions in the groups $B$ and $C$ as multiplication. Then our main results are stated as follows:

THEOREM 1.1. For each integer $m$, let $X_{m}$ be the total space of $S^{2}$-bundle over $S^{4}$ with its characteristic class $\chi\left(X_{m}\right)=m \rho \in \pi_{3}\left(S_{3}\right)$.

If $m$ is a non-zero integer, the sequence

$$
\pi_{6}\left(X_{m}\right) \stackrel{\lambda}{\longrightarrow} E q\left(X_{m}\right) \stackrel{\phi \times \psi}{\longrightarrow} G_{m} \longrightarrow 1
$$

is exact, where

and

$$
G_{m}= \begin{cases}Z_{2} & \text { if }(m, 2)=1 \\ Z_{2} \times Z_{2} & \text { if }(m, 2)=2\end{cases}
$$

$$
\pi_{6}\left(X_{m}\right) \cong \begin{cases}0 & \text { if }(m, 6)=1 \\ Z_{3} & \text { if }(m, 6)=3 \\ Z_{2} \oplus Z_{\left(12, m^{\prime}\right)} & \text { if } m=2 m^{\prime} \text { for some integer } m^{\prime}\end{cases}
$$

Here we denote by $(m, n)$ the greatest common measure of integers $m$ and $n$.

COROLlaRY 1.2. If $(m, 6)=1$, then $E q\left(X_{m}\right) \cong Z_{2}$.

Proposition 1.3. If $(m, 6)=3$, then the group $E q\left(X_{m}\right)$ is isomorphic to $Z_{2}$ or $Z_{6}$ and $\operatorname{Im}\left[\Sigma: E q\left(X_{m}\right) \rightarrow E q\left(\Sigma X_{m}\right)\right] \cong Z_{2}$, where $\Sigma$ denotes the suspension homomorphism.

Remark 1.4. (1) If $m=0, X_{0}=S^{2} \times S^{4}$ and the group $E q\left(X_{0}\right)$ was already well-known. In fact, the following sequence is split exact [17]:

$$
0 \longrightarrow Z_{2} \oplus Z_{2} \longrightarrow E q\left(X_{0}\right) \longrightarrow Z_{2} \times Z_{2} \times Z_{2} \longrightarrow 1
$$

(2) If $m=2 m^{\prime} \neq 0$, the homomorphism $\lambda: \pi_{6}\left(X_{m}\right) \rightarrow E q\left(X_{m}\right)$ is not trivial. In fact, $\operatorname{Im} \lambda$ contains the subgroup isomorphic to $Z_{2}$.

This paper is organized as follows:

In section 2 , we will determine the homotopy groups $\pi_{*}\left(L_{m}\right)$ and $\pi_{*}\left(\Sigma L_{m}\right)$, and in section 3, we will calculate $E q\left(L_{m}\right)$ and $E q\left(\sum L_{m}\right)$. In section 4, we will study the image of the homomorphism $\phi \times \phi$, and in section 5 , we will give the proof of our main results.

\section{Homotopy Groups $\pi_{*}\left(L_{m}\right)$ and $\pi_{*}\left(\Sigma L_{m}\right)$.}

Let $\iota_{n}$ be the oriented generator of $\pi_{n}\left(S^{n}\right)$ and $\eta_{2} \in \pi_{3}\left(S^{2}\right)$ be the Hopf map. 
We put $\eta_{n}=E^{n-2} \eta_{2}, \eta_{n}^{2}=\eta_{n} \circ \eta_{n+1}$ and $\eta_{n}^{3}=\eta_{n} \circ \eta_{n+1} \circ \eta_{n+2}$ for $n>1$, where $E^{n-2}$ denotes the iterated suspension homomorphism. Let $\omega \in \pi_{6}\left(S^{3}\right)$ be the BlackeyMassey element, and $\rho: S^{3} \rightarrow R P^{3}=\mathrm{SO}_{3}$ be the double covering projection. Then the following is well-known:

LEMma 2.1. (H. Toda, [19])

(1) $\pi_{n}\left(S^{n}\right)=Z\left\{\iota_{n}\right\}$ and $\pi_{i}\left(S^{n}\right)=0$ for $i<n$.

(2) $\pi_{n+1}\left(S^{n}\right)=Z_{2}\left\{\eta_{n}\right\}$ for $n>2$ and $\pi_{3}\left(S^{2}\right)=Z\left\{\eta_{2}\right\}$.

(3) $\pi_{n+2}\left(S^{n}\right)=Z_{2}\left\{\eta_{n}^{2}\right\}$ for $n>1$

(4) $\pi_{5}\left(S^{2}\right)=Z_{2}\left\{\eta_{2}^{3}\right\}$ and $\pi_{6}\left(S^{3}\right)=Z_{12}\{\omega\}$

(5) $J(\rho)= \pm \omega$,

where $J: \pi_{3}\left(\mathrm{SO}_{3}\right)=Z\{\rho\} \rightarrow \pi_{6}\left(S^{3}\right)$ denotes the J-homomorphism.

(6) $\eta_{3}^{2}=\left\{2 \iota_{3}, \eta_{3}, 2 \iota_{4}\right\}$ modulo zero,

where $\{,$,$\} denotes the Toda bracket.$

Let $L_{m}$ be the $C W$ complex formed by attaching the 4-cell $e^{4}$ to $S^{2}$ with the map $m \eta_{2} \in \pi_{3}\left(S^{2}\right)$, and $a_{m}:\left(D^{4}, S^{3}\right) \rightarrow\left(L_{m}, S^{2}\right)$ be the characteristic map of the 4cell in $L_{m}$ and $X_{m}$ be the total space of $S^{2}$-bundle over $S^{4}$ with its characteristic element $\chi\left(X_{m}\right)=m \rho \in \pi_{3}\left(S O_{3}=Z\{\rho\}\right.$ for an integer $m$. We denote by the map $p: \mathrm{SO}_{3} \rightarrow \mathrm{SO}_{3} / \mathrm{SO}_{2}=S^{2}$ the natural projection map, and by the map $i: S^{2} \rightarrow L_{m}$ (resp. $i_{1}: L_{m} \rightarrow\left(L_{m}, S^{2}\right)$ ) the inclusion map. Then we have

LEMMA 2.2. (1) $p_{*}(\rho)=\eta_{2}$.

(2) $\pi_{4}\left(L_{m}, S^{2}\right)=Z\left\{a_{m}\right\}$.

(3) $\pi_{5}\left(L_{m}, S^{2}\right)=Z\left\{\left[a_{m}, \iota_{2}\right]_{r}\right\} \oplus a_{m *} \pi_{5}\left(D^{4}, S^{3}\right)$,

where $[,]_{r}$ denotes the relative Whitehead product.

Proof. The statements (1) and (2) are obvious, and the statement (3) follows from (3.1) in [3].

Q.E. D.

Since $p_{*}\left(\chi\left(X_{m}\right)\right)=m \eta_{2}$, we also have

Lemma 2.3. (I. M. James and J. H. C. Whitehead, [5], [6])

(1) The space $X_{m}$ has the $C W$-decomposition $X_{m}=L_{m} \bigcup_{b_{m}} e^{6}$ for some element $b_{m} \in \pi_{5}\left(L_{m}\right)$, and

(2) $i_{1 *}\left(b_{m}\right)=\left[a_{m}, \iota_{2}\right]_{r}$

where $i_{1 *}$ denotes the induced homomorphism 


$$
i_{1 *}: \pi_{5}\left(L_{m}\right) \longrightarrow \pi_{5}\left(L_{m}, S^{2}\right) .
$$

Proof. The above statements follow from (3.3) in [5] and (5.1) in [6].

LEMMA 2.4. (1) $\pi_{1}\left(L_{m}\right)=0$ and $\pi_{2}\left(L_{m}\right)=Z\{i\}$.

(2) $\pi_{3}\left(L_{m}\right)=Z_{m}\left\{i \circ \eta_{2}\right\}$.

(3) $\pi_{4}\left(L_{m}\right)=\left\{\begin{array}{lll}0 & \text { if } m \equiv 1 \quad(\bmod 2) \\ Z_{2}\left\{i \circ \eta_{2}^{2}\right\} & \text { if } m \equiv 0 \quad(\bmod 2) \text { and } m \neq 0 \\ Z\left\{i_{4}\right\} \oplus Z_{2}\left\{i \circ \eta_{2}^{2}\right\} & \text { if } m=0,\end{array}\right.$

where the map $i_{4}: S^{3} \rightarrow S^{2} \vee S^{4}=L_{0}$ denotes the inclusion map to the second factor.

Proof. Without loss of generalities, we may suppose $m \neq 0$ and it suffices only to show the statement (3). Consider the homotopy exact sequence of the pair $\left(L_{m}, S^{2}\right)$ :

$$
\pi_{5}\left(L_{m}, S^{2}\right) \stackrel{\partial_{5}}{\longrightarrow} \pi_{4}\left(S^{2}\right) \stackrel{i_{*}}{\longrightarrow} \pi_{4}\left(L_{m}\right) \stackrel{\imath_{1 *}}{\longrightarrow} \pi_{4}\left(L_{m}, S^{2}\right) \stackrel{\partial_{4}}{\longrightarrow} \pi_{3}\left(S^{2}\right) \longrightarrow \pi_{3}\left(L_{m}\right) \longrightarrow 0 .
$$

Since $\partial_{4}\left(a_{m}\right)=m \eta_{2}$, it follows from (2.2) that the homomorphism $\imath_{1 *}$ is epimorphic. By using the equation $\left[\eta_{2}, \iota_{2}\right]=0, \partial_{5}\left(\left[a_{m}, \iota_{2}\right]_{r}\right)=-\left[\partial_{4}\left(a_{m}\right), \iota_{2}\right]=0$. Similarly,

$$
\begin{aligned}
\partial_{5}\left(a_{m *} \pi_{5}\left(D^{4}, S^{3}\right)\right)= & \left(m \eta_{2}\right)_{*} \pi_{4}\left(S^{3}\right) \\
& =\left\{\begin{array}{lll}
\pi_{4}\left(S^{2}\right) & \text { if } m \equiv 1 & (\bmod 2) \\
0 & \text { if } m \equiv 0 & (\bmod 2) .
\end{array}\right.
\end{aligned}
$$

Hence the assertion (3) easily follows from (2.2).

Q.E. D.

COROLLARY 2.5. If $m \neq 0$, then

$$
\operatorname{Ker}\left[\partial_{5}: \pi_{5}\left(L_{m}, S^{2}\right) \longrightarrow \pi_{4}\left(S^{2}\right)\right]=\left\{\begin{array}{lll}
\pi_{5}\left(L_{m}, S^{2}\right) & \text { if } m \equiv 0 & (\bmod 2) \\
Z\left\{\left[a_{m}, \iota_{2}\right]_{r}\right\} & \text { if } m \equiv 1 & (\bmod 2) .
\end{array}\right.
$$

LEMMA 2.6. If $m \neq 0$, then

$$
\operatorname{Im}\left[\partial_{6}: \pi_{6}\left(L_{m}, S^{2}\right) \longrightarrow \pi_{5}\left(S^{2}\right)\right]=\left\{\begin{array}{lll}
\pi_{5}\left(S^{2}\right) & \text { if } m \equiv 1 & (\bmod 2) \\
0 & \text { if } m \equiv 0 & (\bmod 2) .
\end{array}\right.
$$

Proof. First, we suppose that $m$ is an odd integer. Since $\partial_{6}\left(a_{m *} \pi_{6}\left(D^{4}, S^{3}\right)\right)$ $=\left(m \eta_{2}\right)_{*} \pi_{5}\left(S^{3}\right)=\eta_{2 *} \pi_{5}\left(S^{3}\right)=\pi_{5}\left(S^{2}\right)$, the boundary homomorphism $\partial_{6}$ is epimorphic.

Next, we assume that $m$ is a non-zero even integer. Consider the commutative diagram 


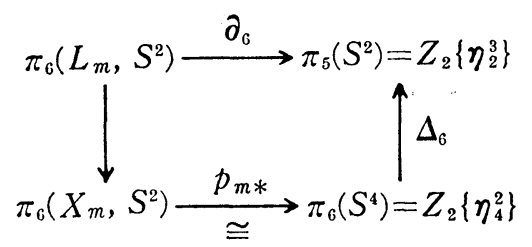

where $\Delta_{n}$ denotes the boundary homomorphism induced from the fibration

$$
S^{2} \longrightarrow X_{m} \stackrel{p_{m}}{\longrightarrow} S^{4}, \Delta_{n}: \pi_{n}\left(S^{4}\right) \longrightarrow \pi_{n-1}\left(S^{2}\right) .
$$

Since $\chi\left(X_{m}\right)=m \rho$, we have

$$
\Delta_{4}\left(\iota_{4}\right)=m \eta_{2} .
$$

Because $\eta_{4}^{2}=E\left(\eta_{3}^{2}\right), \Delta_{6}\left(\eta_{4}^{2}\right)=\Delta_{4}\left(\iota_{4}\right) \circ \eta_{3}^{2}=\left(m \eta_{2}\right) \circ \eta_{3}^{2}=m\left(\eta_{2}^{3}\right)=0$. Hence $\Delta_{6}$ is a trivial homomorphism. Thus the assertion follows from the above diagram. Q. E. D.

Let $q_{m}: L_{m} \rightarrow L_{m} / S^{2}=S^{4}$ be the pinching map which pinches $S^{2}$ in $L_{m}$ to its base point. Then we have

LEMMA 2.8. (S. Oka)

Let $m$ be an even integer. Then there is an element $\gamma_{m} \in \pi_{5}\left(L_{m}\right)$ satısfying the following two conditions:

and

(1) $q_{m} \circ \gamma_{m}=\eta_{4}$

(2) the order of $\gamma_{m}$ is 2 if $m \equiv 0(\bmod 4)$ and 4 if $m \equiv 2(\bmod 4)$.

Proof. We put $m=2 m^{\prime}$. For each positive integer $n$, let $M_{n}$ be the Moore space of type $\left(3, Z_{n}\right), M_{n}=S^{3} \cup_{n \iota_{3}} e^{4}$. Consider the following commutative diagram :

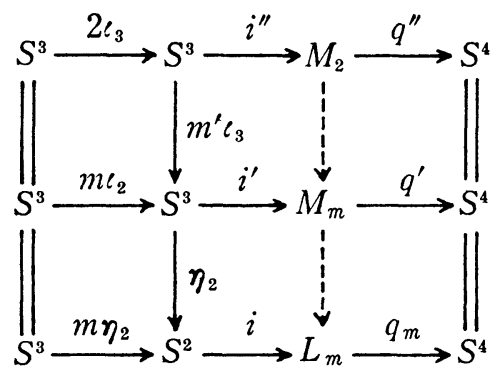

where three horizontal sequences are cofiber sequences. It follows from the above diagram that there are two maps

$$
f: M_{2} \longrightarrow M_{m} \text { and } g: M_{m} \longrightarrow L_{m}
$$


satisfying the following conditions :

$$
q^{\prime} \circ f=q^{\prime \prime}, \quad f \circ i^{\prime \prime}=i^{\prime} \circ\left(m^{\prime} \iota_{3}\right), \quad q_{m} \circ g=q^{\prime}, \text { and } g \circ i^{\prime}=i \circ \eta_{2} .
$$

On the other hand, since $\left(2 \iota_{3}\right) \circ \eta_{3}=\eta_{3} \circ\left(2 \iota_{4}\right)=0$, there is a coextension of $\eta_{3}$, satisfying the condition,

$$
\tilde{\eta}: S^{5} \longrightarrow M_{2}
$$

$$
q^{\prime \prime} \circ \tilde{\eta}=\eta_{4}
$$

From Prop. 1.8 in [19],

Hence we have

$$
\begin{aligned}
2 \tilde{\eta} & =\tilde{\eta} \circ\left(2 \iota_{5}\right) \\
& =i^{\prime \prime} \circ\left\{2 \iota_{3}, \eta_{3}, 2 \iota_{4}\right\} \\
& =i^{\prime \prime} \circ \eta_{3}^{2} \quad(\text { by }(6) \text { in Lemma 2.1) }
\end{aligned}
$$

$$
2 \tilde{\eta}=i^{\prime \prime} \circ \eta_{3}^{2} .
$$

Now we put $\gamma_{m}=g \circ f \circ \tilde{\eta}$. Then,

Similarly,

$$
\begin{array}{rlrl}
q_{m} \circ \gamma_{m} & =q_{m} \circ g \circ f \circ \tilde{\eta} \\
& =q^{\prime \prime} \circ \tilde{\eta} & & (\text { by }(2.9)) \\
& =\eta_{4} & & (\text { by }(2.10))
\end{array}
$$

$$
\begin{array}{rlrl}
2 \gamma_{m} & =g \circ f \circ(2 \tilde{\eta}) & & \\
& =g \circ f \circ\left(i^{\prime \prime} \circ \eta_{3}^{2}\right) & & (\text { by }(2.11)) \\
& =i \circ \eta_{2} \circ\left(m^{\prime} \iota_{3}\right) \eta_{3}^{2} & & (\text { by }(2.9)) \\
& =m^{\prime}\left(i \circ \eta_{2}^{3}\right) & & \\
& =\left\{\begin{array}{lll}
i_{*}\left(\eta_{2}^{3}\right) & \text { if } m^{\prime} \equiv 1 & (\bmod 2) \\
0 & \text { if } m^{\prime} \equiv 0 & (\bmod 2)
\end{array}\right.
\end{array}
$$

Since the order of $i_{*}\left(\eta_{2}^{3}\right)$ is 2 , the order of $\gamma_{m}$ is 4 if $m \equiv 2(\bmod 4)$ and 2 if $m \equiv 0$ $(\bmod 4)$. This completes the proof.

Q.E.D.

Remark. 2.12. The order of the element $\gamma_{m}$ is essentially determined by the suspension order of $S^{2} \bigcup_{m_{c_{2}}} e^{3}$.

Proposition 2.13. (1) If $m \equiv 1(\bmod 2)$, then $\pi_{5}\left(L_{m}\right)=Z\left\{b_{m}\right\}$.

(2) If $m \equiv 0(\bmod 2)$, then

$$
\pi_{5}\left(L_{m}\right)=\left\{\begin{array}{lll}
Z\left\{b_{m}\right\} \oplus Z_{4}\left\{\gamma_{m}\right\} & \text { if } m \equiv 2 & (\bmod 4) \\
Z\left\{b_{m}\right\} \oplus Z_{2}\left\{\gamma_{m}\right\} \oplus Z_{2}\left\{\imath \circ \eta_{2}^{3}\right\} & \text { if } m \equiv 0 & (\bmod 4)
\end{array}\right.
$$


where we can put $b_{m}=\left[i, i_{4}\right]$ and $\gamma_{m}=i_{4} \circ \eta_{4}$ if $m=0$, and $2 \gamma_{m}=i \circ \eta_{2}^{2}$ if $m \equiv 2$ $(\bmod 4)$.

Proof. Consider the homotopy exact sequence of the pair $\left(L_{m}, S^{2}\right)$,

$$
\pi_{6}\left(L_{m}, S^{2}\right) \stackrel{\partial_{6}}{\longrightarrow} \pi_{5}\left(S^{2}\right) \stackrel{i_{*}}{\longrightarrow} \pi_{5}\left(L_{m}\right) \stackrel{i_{1 *}}{\longrightarrow} \pi_{5}\left(L_{m}, S^{2}\right) \stackrel{\partial_{5}}{\longrightarrow} \pi_{4}\left(S^{2}\right) .
$$

First, we suppose that $m \equiv 1(\bmod 2)$. Then it follows from $(2.3),(2.5)$ and $(2.6)$ that we have $\pi_{5}\left(L_{m}\right)=Z\left\{b_{m}\right\}$. If $m=0$, then $L_{0}=S^{2} \vee S^{4}$ and the assertion clearly holds. Hence, we assume $m \equiv 0(\bmod 2)$ and $m \neq 0$. Then, from $(2.3)$, (2.5) and (2.6) we have the following results :

(1) $\pi_{5}\left(L_{m}\right)=Z\left\{b_{m}\right\} \oplus \operatorname{Tor}\left(\pi_{5}\left(L_{m}\right)\right)$.

(2) The sequence

$$
0 \longrightarrow \pi_{5}\left(S^{2}\right) \stackrel{i_{*}}{\longrightarrow} \operatorname{Tor}\left(\pi_{5}\left(L_{m}\right)\right) \stackrel{\imath_{1 *}}{\longrightarrow} a_{m *} \pi_{5}\left(D^{4}, S^{3}\right) \longrightarrow 0
$$

is exact. On the other hand, it follows from Theorem 2.1 in [2] that the sequence

$$
0 \longrightarrow \pi_{2}\left(S^{2}\right) \stackrel{Q}{\longrightarrow} \pi_{5}\left(L_{m}, S^{2}\right) \stackrel{q_{m *}}{\longrightarrow} \pi_{5}\left(S^{4}\right) \longrightarrow 0
$$

is exact, where the homomorphism $Q$ is defined by the relative Whitehead product, $Q\left(\iota_{2}\right)=\left[a_{m}, \iota_{2}\right]_{r}$. Hence the map $q_{m}$ induces the isomorphism $q_{m *}$ : $a_{m *} \pi_{5}\left(D^{4}, S^{3}\right) \stackrel{\cong}{\longrightarrow} \pi_{5}\left(S^{4}\right)$, and we have the following exact sequence,

$$
0 \longrightarrow \pi_{5}\left(S^{2}\right) \stackrel{i_{*}}{\longrightarrow} \operatorname{Tor}\left(\pi_{5}\left(L_{m}\right)\right) \stackrel{q_{m *}}{\longrightarrow} \pi_{5}\left(S^{4}\right) \longrightarrow 0 .
$$

Hence it follows from (2.8) and (2.15) that we have

$$
\operatorname{Tor}\left(\pi_{5}\left(L_{m}\right)\right)=\left\{\begin{array}{lll}
Z_{4}\left\{\gamma_{m}\right\} & \text { if } m \equiv 2 & (\bmod 4) \\
Z_{2}\left\{\gamma_{m}\right\} \oplus Z_{2}\left\{i \circ \eta_{2}^{3}\right\} & \text { if } m \equiv 0 & (\bmod 4) .
\end{array}\right.
$$

Therefore, we obtain the desired results.

Q.E. D.

In the rest of this section, we will consider the homotopy group $\pi_{*}\left(\Sigma L_{m}\right)$. First, we remark that

$$
\Sigma L_{m}=\left\{\begin{array}{lll}
S^{3} \vee S^{5} & \text { if } m \equiv 0 & (\bmod 2) \\
\Sigma C P^{2} & \text { if } m \equiv 1 & (\bmod 2)
\end{array}\right.
$$

For each even integer $m$, let $i_{5}: S^{5} \rightarrow \Sigma L_{m}=S^{3} \vee S^{5}$ denote the inclusion map to the second factor.

LEMMA 2.18. Let $m$ be an even integer. Then thesusp ension homomorphism 


$$
\Sigma: \operatorname{Tor}\left(\pi_{5}\left(L_{m}\right)\right) \longrightarrow \pi_{6}\left(\Sigma L_{m}\right)=\pi_{6}\left(S^{3} \vee S^{5}\right)
$$

is injective, where $\pi_{6}\left(\Sigma L_{m}\right)=\pi_{6}\left(S^{3} \vee S^{5}\right)=Z_{12}\{(\Sigma i) \circ \omega\} \oplus Z_{2}\left\{i_{5} \circ \eta_{5}\right\}$ and we can choose the map $\gamma_{m} \in \operatorname{Tor}\left(\pi_{5}\left(L_{m}\right)\right)$ to satisfy the condition

$$
\Sigma \gamma_{m}=\left\{\begin{array}{lll}
i_{5} \circ \eta_{5} & \text { if } m \equiv 0(\bmod 4) \\
i_{5} \circ \eta_{5}+3(\Sigma i) \circ \omega & \text { if } m \equiv 2(\bmod 4) .
\end{array}\right.
$$

Remark 2.20. It is easy to see that there are two possibilities of the choice of $\gamma_{m}, \gamma_{m}$ and $\gamma_{m}+i_{*}\left(\eta_{2}^{3}\right)$. However, if $\gamma_{m}$ satisfies the condition (2.19), then it is uniquely determined.

Proof. Since $\Sigma L_{m}=S^{3} \vee S^{5}$, we have the following commutative diagram:

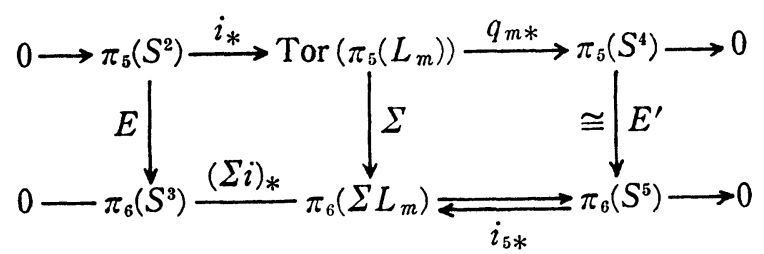

where $E, E^{\prime}$ and $\Sigma$ denote the suspension homomorphisms. Since $E$ is monic and $E^{\prime}$ is isomorphic, it follows from the five Lemma that $\Sigma$ is also monomorphic. Hence the order of $\Sigma \gamma_{m}$ is 2 if $m \equiv 0(\bmod 4)$ and 4 if $m \equiv 2(\bmod 4)$. Then it follows from $q_{m *}\left(\gamma_{m}\right)=\eta_{4}$ that we have $\Sigma \gamma_{m} \equiv i_{5} \circ \eta_{5}$ modulo $\operatorname{Im}(\Sigma i)_{*}$. Therefore, there exists some integer $n$ satisfying the condition

$$
\Sigma \gamma_{m}=\left\{\begin{array}{lll}
i_{5} \circ \eta_{5}+n(\Sigma i) \circ \eta_{3}^{3} & \text { if } m \equiv 0 \quad(\bmod 4) \\
i_{5} \circ \eta_{5}+3(\Sigma i) \circ \omega+n(\Sigma i) \circ \eta_{3}^{3} & \text { if } m \equiv 2(\bmod 4) .
\end{array}\right.
$$

Then by using the base change $\gamma_{m} \rightarrow \gamma_{m}+n\left(i \circ \eta_{2}^{3}\right)$, it is easy to see that $\gamma_{m}$ satisfies the condition (2.19).

Q.E. D.

An easy calculation shows the following results, and we will omit the proof.

LEMMA 2.21. Let $m$ be an odd integer. Then we have the following results.

(1) $\pi_{i}\left(\Sigma L_{m}\right)=0$ for $i=1,2$ or 4 ,

(2) $\pi_{3}\left(\Sigma L_{m}\right)=Z\{\Sigma i\}$

(3) $\pi_{5}\left(\Sigma L_{m}\right)=Z\left\{\widetilde{2 \iota_{4}}\right\}$, and

(4) $\pi_{6}\left(\Sigma L_{m}\right)=Z_{6}\{(\Sigma i) \circ \omega\}$,

where we denote by $\widetilde{2 \iota_{4}}$ the coextension of $2 \iota_{4}$ which satisfies the condition $\left(\sum q_{m}\right) \circ \widetilde{2 \iota_{4}}$ $=2 \iota_{5}$. 
3. The Groups $E q\left(L_{m}\right)$ and $E q\left(\Sigma L_{m}\right)$.

In this section, we will determine the group structure of $E q\left(L_{m}\right)$ and $E q\left(\Sigma L_{m}\right)$.

Definition 3.1. Let $K$ be a $C W$ complex with $\operatorname{dim} K<n$. Let $X$ be a $C W$ complex formed by attaching the $n$-cell $e^{n}$ to $K$ with the map $f \in \pi_{n-1}(K)$, $X=K \bigcup_{f} e^{n}$. We denote by $i, \mu$ and $\nabla$ the inclusion map, a co-action map and a folding map, respectively. Then we define the homomorphism $\lambda: \operatorname{Im}\left[i_{*}: \pi_{n}(K)\right.$ $\left.\rightarrow \pi_{n}(X)\right] \rightarrow E q(X)$ by the following:

$$
\lambda(i \circ g)=\nabla \circ\left(i d_{X} \vee i \circ g\right) \circ \mu: X \stackrel{\mu}{\longrightarrow} X \vee S^{n} \stackrel{i d_{X} \vee \imath \circ g}{\longrightarrow} X \vee X \stackrel{\nabla}{\longrightarrow} X \text { for } g \in \pi_{n}(K) .
$$

Similarly, we define two homomorphisms

$$
\phi: E q(X) \longrightarrow E q(K) \text { and } \phi: E q(X) \longrightarrow E q\left(S^{n}\right)=Z_{2}
$$

by the restriction of maps and the degree of the top cell $e^{n}$. (See in detail, [12])

Secondly, we construct the elements of $E q\left(L_{m}\right)$ and $E q\left(\Sigma L_{m}\right)$.

Definition 3.2. (1) For $m=0$, we define the map $h_{0}$ by the equation,

$$
h_{0}=\iota_{2} \vee\left(-\iota_{4}\right): L_{0}=S^{2} \vee S^{4} \longrightarrow L_{0}=S^{2} \vee S^{4} \text {. }
$$

For each even integer $m$, we define the map $h_{0}^{\prime}$ by the equation

$$
h_{0}^{\prime}=\iota_{3} \vee\left(-\iota_{5}\right): \Sigma L_{m}=S^{3} \vee S^{5} \longrightarrow \Sigma L_{m}=S^{3} \vee S^{5} \text {. }
$$

Clearly, if $m=0, h_{0}^{\prime}=\Sigma h_{0}$. For each odd integer $m$, let $h_{0}^{\prime}: \Sigma L_{m} \rightarrow \Sigma L_{m}$ be one of the maps which has a degree +1 on $S^{3}$ and -1 on the cell $e^{5}$ in $\Sigma L_{m}$. Since $\eta_{3}=-\eta_{3}$, the map $h_{0}^{\prime}$ always exists.

(2) For each integer $m$, let $h_{1}: L_{m} \rightarrow L_{m}$ be one of the maps which has a degree -1 on $S^{2}$ and a degree +1 on the cell $e^{4}$ in $L_{m}$. Since

$$
\begin{aligned}
& H\left(\eta_{2}\right)=\iota_{3} \text { and }\left[\iota_{2}, \iota_{2}\right]=2 \eta_{2}, \\
& \begin{aligned}
\left(-\iota_{2}\right) \circ\left(m \eta_{2}\right) & =m\left(\left(-\iota_{2}\right) \circ \eta_{2}\right) \\
& =m\left(-\eta_{2}+\left[\iota_{2}, \iota_{2}\right] \cdot H\left(\eta_{2}\right)\right)=m \eta_{2} .
\end{aligned}
\end{aligned}
$$

Hence the map $h_{1}$ always exists. We define the map $h_{1}^{\prime}: \Sigma L_{m} \rightarrow \Sigma L_{m}$ by the equation

$$
h_{1}^{\prime}=\left\{\begin{array}{lll}
\left(-\iota_{3}\right) \vee \iota_{5}: \Sigma L_{m}=S^{3} \vee S^{5} \rightarrow \Sigma L_{m}=S^{3} \vee S^{5} & \text { if } m \equiv 0 & (\bmod 2) \\
\Sigma h_{1}: \Sigma L_{m} \rightarrow \Sigma L_{m} & \text { if } m \equiv 1 & (\bmod 2) .
\end{array}\right.
$$

(3) For each integer $m$, we define the map $h_{2}$ by the equation $h_{2}=\lambda\left(\eta_{2}^{2}\right)$. 
In particular, if $m=0$, then $h_{2}=i d_{L_{0}}+i \circ \eta_{2}^{2} \circ p r$, where $p r$ denotes the projection map to the second factor, $p r: L_{0}=S^{2} \vee S^{4} \rightarrow S^{4}$. For each even integer $m$, we define the map $h_{2}^{\prime}: \Sigma L_{m} \rightarrow \Sigma L_{m}$ by the equation $h_{2}^{\prime}=\Sigma h_{2}$. Then it is easy to see that $h_{2}^{\prime}=i d_{\Sigma L_{m}}+(\Sigma i) \circ \eta_{3}^{2} \circ(\Sigma p r)$.

THEOREM 3.3.

(1) $E q\left(L_{m}\right)=\left\{\begin{array}{lll}Z_{2}\left\{h_{1}\right\} & \text { if } m \equiv 1 \quad(\bmod 2) \\ Z_{2}\left\{h_{1}\right\} \times Z_{2}\left\{h_{2}\right\} & \text { if } m \equiv 0 \quad(\bmod 2) \text { and } m \neq 0 \\ Z_{2}\left\{h_{0}\right\} \times Z_{2}\left\{h_{1}\right\} \times Z_{2}\left\{h_{2}\right\} & \text { if } m=0 .\end{array}\right.$

(2) $E q\left(\Sigma L_{m}\right)=\left\{\begin{array}{lll}Z_{2}\left\{h_{0}^{\prime}\right\} \times Z_{2}\left\{h_{1}^{\prime}\right\} & \text { if } m \equiv 1 \quad(\bmod 2) \\ Z_{2}\left\{h_{0}^{\prime}\right\} \times Z_{2}\left\{h_{1}^{\prime}\right\} \times Z_{2}\left\{h_{2}^{\prime}\right\} & \text { if } m \equiv 0 & (\bmod 2) .\end{array}\right.$

(3) The suspension homomorphism $\Sigma: E q\left(L_{m}\right) \rightarrow E q\left(\Sigma L_{m}\right)$ is monomorphic and isomorphic if $m=0$.

Proof. First, we consider the case $m=0$. Since $L_{0}=S^{2} \vee S^{4}$, the homotopy set $\left[L_{0}, L_{0}\right]$ has a natural ring structure which is induced from the track addition and the composition of maps. It is easy to see that the ring $\left[L_{0}, L_{0}\right]$ is isomorphic to the matrix ring

$$
\left[\begin{array}{cc}
\pi_{2}\left(S^{2}\right) & \pi_{4}\left(S^{2}\right) \\
\pi_{2}\left(S^{4}\right) & \pi_{4}\left(S^{4}\right)
\end{array}\right]=\left[\begin{array}{cc}
Z\left\{\iota_{2}\right\} & Z_{2}\left\{\eta_{2}^{2}\right\} \\
0 & Z\left\{\iota_{4}\right\}
\end{array}\right]
$$

Hence $E q\left(L_{0}\right)=\left\{ \pm h_{0}, \pm h_{1}, \pm h_{2}\right\}$, where $h_{0}=\iota_{2} \vee\left(-\iota_{4}\right)=\left[\begin{array}{cc}1 & 0 \\ 0 & -1\end{array}\right]$,

$$
h_{1}=\left(-\iota_{2}\right) \vee \iota_{4}=\left[\begin{array}{cc}
-1 & 0 \\
0 & 1
\end{array}\right] \text { and } h_{2}=i d_{L_{0}}+i \circ \eta_{2}^{2} \circ p r=\left[\begin{array}{cc}
1 & \eta_{2}^{2} \\
0 & 1
\end{array}\right] \text {. }
$$

Since $h_{n} \circ h_{n}=\imath d_{L_{0}}$ and $h_{n} \circ h_{k}=h_{k} \circ h_{n}$ for $0 \leqq n, k \leqq 2$, we have

$$
E q\left(L_{0}\right)=Z_{2}\left\{h_{0}\right\} \times Z_{2}\left\{h_{1}\right\} \times Z_{2}\left\{h_{2}\right\} .
$$

A similar argument shows $E q\left(\Sigma L_{0}\right)=Z_{2}\left\{h_{0}^{\prime}\right\} \times Z_{2}\left\{h_{1}^{\prime}\right\} \times Z_{2}\left\{h_{2}^{\prime}\right\}$, and it follows from $h_{n}^{\prime}=\Sigma h_{n}$ that the suspension homomorphism $\Sigma: E q\left(L_{0}\right) \rightarrow E q\left(\Sigma L_{0}\right)$ is isomorphic.

Next, we consider the case $m \neq 0$. It follows from the Barcus-Barratt theorem [2] and Theorem 3.13 in [12] that we obtain the following commutative diagram:

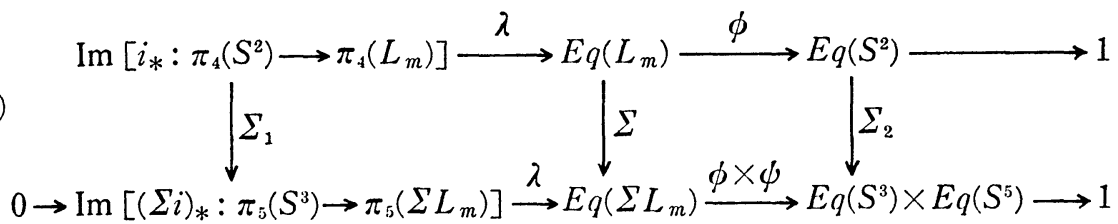


where $\Sigma, \Sigma_{1}$ and $\Sigma_{2}$ denote the suspension homomorphisms, and two horizontal sequences are exact.

Now we suppose that $m$ is an odd integer. Since $\pi_{4}\left(L_{m}\right)=\operatorname{Im}(\Sigma i)_{*}=0$ and $\Sigma_{2}$ is monomorphic, $E q\left(L_{m}\right)=Z_{2}\left\{h_{1}\right\}, E q\left(\Sigma L_{m}\right)=Z_{2}\left\{h_{0}^{\prime}\right\} \times Z_{2}\left\{h_{1}^{\prime}\right\}$ and $\Sigma$ is a monomorphism.

Finally we assume that $m$ is a non-zero even integer. Since $\Sigma L_{m}=S^{3} \vee S^{5}$, we obtain

$$
E q\left(\Sigma L_{m}\right)=Z_{2}\left\{h_{0}^{\prime}\right\} \times Z_{2}\left\{h_{1}^{\prime}\right\} \times Z_{2}\left\{h_{2}^{\prime}\right\} \text {, and } \operatorname{Im}(\Sigma i)_{*}=Z_{2}\left\{(\Sigma i) \circ \eta_{3}^{2}\right\} \text {. }
$$

Hence $\Sigma_{1}$ is an isomorphism and the homomorphism $\lambda: \operatorname{Im} i_{*}=Z_{2}\left\{i \circ \eta_{2}^{2}\right\} \rightarrow E q\left(L_{m}\right)$ is a monomorphism. Because $\Sigma_{2}$ is monic, according to the Five Lemma, $\Sigma$ is a monomorphism. Therefore, it follows from (3.5) that $E q\left(L_{m}\right)=Z_{2}\left\{h_{1}\right\} \times Z_{2}\left\{h_{2}\right\}$.

Q.E.D.

\section{The Image of the Homomorphism $\phi \times \psi$.}

The purpose of this section is to determine the image of $\phi \times \phi$,

$$
G_{m}=\operatorname{Im}\left[\phi \times \phi: E q\left(X_{m}\right) \longrightarrow E q\left(L_{m}\right) \times E q\left(S^{6}\right)\right] .
$$

According to Lemma 2.2 in [12], if we identify $E q\left(S^{6}\right)=Z_{2}=\{ \pm 1\}$,

LEMMA 4.2. $G_{m}=\left\{(h, \varepsilon) \in E q\left(L_{m}\right) \times\{ \pm 1\}: h \circ b_{m}=\varepsilon b_{m}\right\}$.

Thus it suffices only to determine the action of $E q\left(L_{m}\right)$ to the homotopy group $\pi_{5}\left(L_{m}\right)$ which is induced from the composition of maps,

$$
E q\left(L_{m}\right) \times \pi_{5}\left(L_{m}\right) \longrightarrow \pi_{5}\left(L_{m}\right) .
$$

Let $j^{\prime}: L_{m} \rightarrow L_{m} \vee S^{4}$ and $j^{\prime \prime}: S^{4} \rightarrow L_{m} \vee S^{4}$ denote the inclusion maps to the first factor and second factor, respectively. Let $\mu: L_{m} \rightarrow L_{m} \vee S^{4}$ be a co-action map. First, we note the following

LEMMA 4.4. (I. M. James, [3])

(1) $\mu_{*}\left(b_{m}\right)=j^{\prime} \circ b_{m}+\left[i, j^{\prime \prime}\right]$.

(2) $\mu_{*}\left(\gamma_{m}\right)=j^{\prime} \circ \gamma_{m}+j^{\prime} \circ \eta_{4}$.

(3) $\mu_{*}\left(i_{*}\left(\eta_{2}^{3}\right)\right)=j^{\prime} \circ i_{*}\left(\eta_{2}^{3}\right)$.

Proof. Let $\eta^{\prime}$ be the generator of $\pi_{5}\left(D^{4}, S^{3}\right) \cong Z_{2}$. According to (2.3), (2.8) and (2.13), $i_{1 *}\left(b_{m}\right)=\left[a_{m}, \iota_{2}\right]_{r}, i_{1 *}\left(\gamma_{m}\right)=a_{m *}\left(\eta^{\prime}\right)$ and $i_{1 *}\left(i_{*}\left(\eta_{2}^{3}\right)\right)=0$. Then the above results follow from Lemma 5.4 in [3].

Q.E.D.

LemMA 4.5. (I. M. James, [4]) $\Sigma b_{m}=m\left(\left(\sum i\right) \circ \omega\right)$.

Proof. Since $J\left(\chi\left(X_{m}\right)\right)=J(m \rho)= \pm m \omega$, the assertion follows from (3.1) in [4].

Q.E. D. 
Then the action (4.3) is described as follows:

THEOREM 4.6. (1) If $m \equiv 1(\bmod 2)$, then $h_{1} \circ b_{m}=-b_{m}$.

(2) If $m \equiv 0(\bmod 2)$, then
(a) $h_{1} \circ b_{m}=-b_{m}$,
(b) $h_{2} \circ b_{m}=b_{m}$,
(c) $h_{1} \circ \gamma_{m}=\left\{\begin{array}{lll}\gamma_{m}+i_{*}\left(\eta_{2}^{3}\right) & \text { if } m \equiv 2(\bmod 4) \text {, } \\ \gamma_{m} & \text { if } m \equiv 0(\bmod 4) \text {, }\end{array}\right.$
(d) $h_{2} \circ \gamma_{m}=\gamma_{m}+i_{*}\left(\eta_{2}^{3}\right)$,

and

(e) $h_{1} \circ i_{*}\left(\eta_{2}^{3}\right)=h_{2} \circ i_{*}\left(\eta_{2}^{3}\right)=i_{*}\left(\eta_{2}^{3}\right)$ if $m \equiv 0(\bmod 4)$.

(3) In particular, if $m=0$, then
(a) $h_{0} \circ b_{0}=-b_{0}$,
(b) $h_{0} \circ \gamma_{0}=\gamma_{0}$,

and

(c) $h_{0} \circ i_{*}\left(\eta_{2}^{3}\right)=i_{*}\left(\eta_{2}^{3}\right)$.

Proof. According to the cellular aproximation theorem, we may assume $h_{1}\left(S^{2}\right) \subset S^{2}$. Hence $h_{1} \circ i_{1}=i_{1} \circ h_{1}$. Therefore,

$$
\begin{aligned}
i_{1 *}\left(h_{1} \circ b_{m}\right) & =h_{1} \circ i_{1 *}\left(b_{m}\right) \\
& =h_{1 *}\left(\left[a_{m}, \iota_{2}\right]_{r}\right) \quad(\text { by }(2.3)) \\
& =\left[h_{1} \circ a_{m},\left(h_{1} \mid S^{2}\right) \circ \iota_{2}\right]_{r} \\
& =\left[a_{m},-\iota_{2}\right]_{r} \\
& =-\left[a_{m}, \iota_{2}\right]_{r} \\
& =-i_{1 *}\left(b_{m}\right) .
\end{aligned}
$$

Thus, we have

$$
h_{1} \circ b_{m}=-b_{m} \quad \text { modulo } i_{*} \pi_{5}\left(S^{2}\right) \text {. }
$$

(1) First, we suppose $m \equiv 1(\bmod 2)$, According to (2.13), $i_{*} \pi_{5}\left(S^{2}\right)=0$ and we have $h_{1} \circ b_{m}=-b_{m}$.

(2) Next, we assume $m \equiv 0(\bmod 2)$.

(a) Since $i_{*} \pi_{5}\left(S^{2}\right)$ is contained in Tor $\left(\pi_{5}\left(L_{m}\right)\right)$, there exists some element $\gamma \in \operatorname{Tor}\left(\pi_{5}\left(L_{m}\right)\right)$ such that $h_{1} \circ b_{m}=-b_{m}+\gamma$. Then 


$$
\begin{aligned}
\Sigma \gamma & =\Sigma\left(h_{1} \circ b_{m}+b_{m}\right) \\
& =\left(\Sigma h_{1}\right) \circ \Sigma b_{m}+\Sigma b_{m} \\
& =h_{1}^{\prime} \circ(m(\Sigma i \circ \omega))+m(\Sigma \imath \circ \omega) \quad(\text { by }(4.5)) \\
& =-m\left(\sum i \circ \omega\right)+m(\Sigma i \circ \omega) \\
& =0 .
\end{aligned}
$$

Therefore, according to (2.18), $\gamma=0$. Hence $h_{1} \circ b_{m}=-b_{m}$.

(b) Since $\left[\iota_{2}, \eta_{2}^{2}\right]=0$,

$$
\begin{aligned}
h_{2} \circ b_{m} & =\lambda\left(\eta_{2}^{2}\right) \circ b_{m} \\
& =\nabla \circ\left(i d_{L_{m}} \vee i \circ \eta_{2}^{2}\right) \circ \mu_{*}\left(b_{m}\right) \\
& =\nabla \circ\left(i d_{L_{m}} \vee i \circ \eta_{2}^{2}\right) \circ\left(j^{\prime} \circ b_{m}+\left[i, j^{\prime \prime}\right]\right) \quad(\text { by }(4.4)) \\
& =b_{m}+i \circ\left[c_{2}, \eta_{2}^{2}\right] \\
& =b_{m} .
\end{aligned}
$$

(c) If $m \equiv 2(\bmod 4)$, then

$$
\begin{aligned}
\Sigma\left(h_{1} \circ \gamma_{m}\right) & =h_{1}^{\prime} \circ \Sigma \gamma_{m} \\
& =\left(\left(-\iota_{3}\right) \vee \iota_{5}\right) \circ\left(i_{5} \circ \eta_{5}+3\left(\Sigma_{\imath} \circ \omega\right)\right) \quad(\text { by }(2.19) \\
& =-\left(i_{5} \circ \eta_{5}+3\left(\Sigma_{\imath} \circ \omega\right)\right) \\
& =-\Sigma \gamma_{m} \\
& =\Sigma\left(\gamma_{m}+i_{*}\left(\eta_{2}^{3}\right)\right) .
\end{aligned}
$$

Hence, according to (2.18), we have $h_{1} \circ \gamma_{m}=\gamma_{m}+i_{*}\left(\eta_{2}^{3}\right)$. If $m=0(\bmod 4)$, then it follows from (2.19) that $\Sigma \gamma_{m}=i_{5} \circ \eta_{5}$. Hence, a similar calculation shows $h_{1} \circ \gamma_{m}=\gamma_{m}$.

(d) $h_{2} \circ \gamma_{m}=\lambda\left(\eta_{2}^{2}\right) \circ \gamma_{m}$

$$
\begin{aligned}
& =\nabla \circ\left(i d_{L_{m}} \vee \imath \circ \eta_{2}^{2}\right) \circ \mu_{*}\left(\gamma_{m}\right) \\
& =\nabla \circ\left(i d_{L_{m}} \vee \imath \circ \eta_{2}^{2}\right) \circ\left(j^{\prime} \circ \gamma_{m}+j^{\prime \prime} \circ \eta_{4}\right) \quad(\text { by }(4.4)) \\
& =\gamma_{m}+\imath_{*}\left(\eta_{2}^{3}\right)
\end{aligned}
$$

(e) $\Sigma\left(h_{1} \circ{ }_{*}\left(\eta_{2}^{3}\right)\right)=h_{1}^{\prime} \circ(\Sigma i) \circ \eta_{3}^{3}$

$$
\begin{aligned}
& =\left(\left(-\iota_{3}\right) \vee \iota_{5}\right) \circ(\Sigma i) \circ \eta_{3}^{3} \\
& =-\Sigma l \circ \eta_{3}^{3} \\
& =\Sigma\left(i \circ \eta_{2}^{3}\right) .
\end{aligned}
$$


Hence, according to (2.18), we have $h_{1} \circ i_{*}\left(\eta_{2}^{3}\right)=i_{*}\left(\eta_{2}^{3}\right)$.

Thus,

$$
\begin{aligned}
h_{2} \circ i_{*}\left(\eta_{2}^{3}\right) & =\nabla \circ\left(i d_{L_{m}} \vee i \circ \eta_{2}^{2}\right) \circ \mu_{*}\left(i_{*}\left(\mu_{2}^{3}\right)\right) \\
& =\nabla \circ\left(i d_{L_{m}} \vee i \circ \eta_{2}^{2}\right) \circ j^{\prime} \circ i_{*}\left(\eta_{2}^{3}\right) \\
& =i_{*}\left(\eta_{2}^{3}\right) .
\end{aligned}
$$

$$
h_{1}{ }^{\circ}{ }_{*}\left(\eta_{2}^{3}\right)=h_{2} \circ i_{*}\left(\eta_{2}^{3}\right)=i_{*}\left(\eta_{2}^{3}\right) .
$$

(3) If $m=0$, then it follows from (2.13) and (3.2) that $h_{0}=\iota_{2} \vee\left(-\iota_{4}\right), \quad b_{0}=$ $\left[i, i_{4}\right]$ and $\gamma_{0}=i_{4} \circ \eta_{4}$. Therefore, it is easy to see the assertion (3) and we omit the proof.

Q.E.D.

In particular, it follows from (4.2) that we obtain the following

COROLLARY 4.8.

$$
G_{m} \cong\left\{\begin{array}{lll}
Z_{2} & \text { if } m \equiv 1 \quad(\bmod 2) \\
Z_{2} \times Z_{2} & \text { if } m \equiv 0 \quad(\bmod 2) \text { and } m \neq 0 \\
Z_{2} \times Z_{2} \times Z_{2} & \text { if } m=0 .
\end{array}\right.
$$

\section{The Proof of the Main Results.}

In this section, we will prove (1.1) and (1.3).

Let $j: L_{m} \rightarrow X_{m}$ be an inclusion map and $b_{m}^{\prime} \in \pi_{6}\left(X_{m}, L_{m}\right)$ denote the characteristic map of the top cell $e^{6}$ in $X_{m}$. Consider the homotopy exact sequence

$$
\pi_{6}\left(L_{m}\right) \stackrel{j_{*}}{\longrightarrow} \pi_{6}\left(X_{m}\right) \longrightarrow \pi_{6}\left(X_{m}, L_{m}\right)=Z\left\{b_{m}^{\prime}\right\} \stackrel{\partial}{\longrightarrow} \pi_{5}\left(L_{m}\right)=Z\left\{b_{m}\right\} \oplus \operatorname{Tor}\left(\pi_{5}\left(L_{m}\right)\right)
$$

Since $\pi_{6}\left(X_{m}, L_{m}\right)=Z\left\{b_{m}^{\prime}\right\}$ and $\partial\left(b_{m}^{\prime}\right)=b_{m}$, we have

LEMMA 5.1. $\operatorname{Im}\left[j_{*}: \pi_{6}\left(L_{m}\right) \longrightarrow \pi_{6}\left(X_{m}\right)\right]=\pi_{6}\left(X_{m}\right)$.

Hence, according to the Barcus-Barratt Theorem and (4.8), we obtain

LEMMA 5.2. The sequence

is exact.

$$
\pi_{6}\left(X_{m}\right) \stackrel{\lambda}{\longrightarrow} E q\left(X_{m}\right) \stackrel{\phi \times \phi}{\longrightarrow} G_{m} \longrightarrow 1
$$

Therefore, to prove (1.1), it suffices only to show the following

PROPOSITION 5.3.

$$
\pi_{6}\left(X_{m}\right) \cong \begin{cases}0 & \text { if }(m, 6)=1 \\ Z_{3} & \text { if }(m, 6)=3 \\ Z_{2} \oplus Z_{\left(12, m^{\prime}\right)} & \text { if } m=2 m^{\prime} \neq 0 .\end{cases}
$$


Consider the homotopy exact sequence of the fibration $\xi_{m}: S^{2} \stackrel{j \circ i}{\longrightarrow} X_{m} \stackrel{p_{m}}{\longrightarrow} S^{4}$,

$$
\pi_{7}\left(S^{4}\right) \stackrel{\Delta_{7}}{\longrightarrow} \pi_{6}\left(S^{2}\right) \longrightarrow \pi_{6}\left(X_{m}\right) \stackrel{p_{m *}}{\longrightarrow} \pi_{6}\left(S^{4}\right) \stackrel{\Delta_{6}}{\longrightarrow} \pi_{5}\left(S^{2}\right) .
$$

Here, according to [19], we note

$$
\begin{aligned}
& \pi_{5}\left(S^{2}\right)=Z_{2}\left\{\eta_{2}^{3}\right\}, \quad \pi_{6}\left(S^{2}\right)=Z_{12}\left\{\eta_{2} \circ \omega\right\}, \quad \pi_{6}\left(S^{4}\right)=Z_{2}\left\{\eta_{4}^{2}\right\}, \\
& \text { and } \pi_{7}\left(S^{4}\right)=Z\left\{\nu_{4}\right\} \oplus Z_{12}\{E \omega\} .
\end{aligned}
$$

Then we have

LEMMA 5.6.

(1) $\Delta_{6}\left(\eta_{4}^{2}\right)=m\left(\eta_{2}^{3}\right)=\left\{\begin{array}{lll}\eta_{2}^{3} & \text { if } m \equiv 1 \quad(\bmod 2) \\ 0 & \text { if } m \equiv 0 & (\bmod 2) \text {. }\end{array}\right.$

(2) $\Delta_{7}(E \boldsymbol{\omega})=m\left(\eta_{2} \circ \boldsymbol{\omega}\right)$.

(3) $\Delta_{7}\left(\nu_{4}\right)= \pm(m(m-1) / 2)\left(\eta_{2} \circ \boldsymbol{\omega}\right)$.

Proof. (1) Since $\eta_{4}^{2}=E\left(\eta_{3}^{2}\right)$, according to (2.7),

$$
\Delta_{6}\left(\eta_{4}^{2}\right)=\Delta_{4}\left(\ell_{4}\right) \circ \eta_{3}^{2}=\left(m \eta_{2}\right) \circ \eta_{3}^{2}=m\left(\eta_{2}^{3}\right) .
$$

(2) Similarly, since $\left[\iota_{3}, \iota_{3}\right]=0, \Delta_{7}(E \boldsymbol{\omega})=\Delta_{4}\left(\iota_{4}\right) \circ \boldsymbol{\omega}=\left(m \eta_{2}\right) \circ \boldsymbol{\omega}=m\left(\eta_{2} \circ \boldsymbol{\omega}\right)$.

(3) Consider the induced fibration $\nu_{4}^{*} \xi_{m}$ :

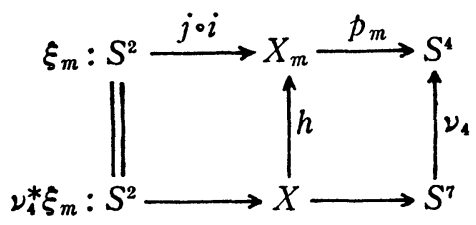

Then we have the commutative diagram

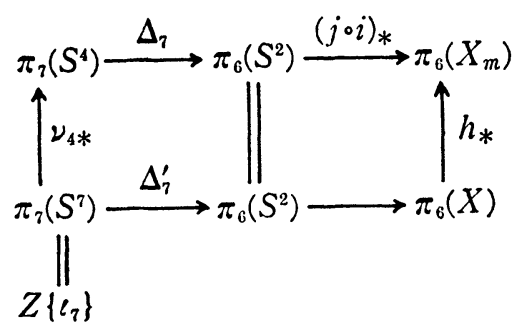

Hemce 


$$
\Delta_{7}\left(\nu_{4}\right)=\Delta_{7}\left(\nu_{4 *}\left(\iota_{7}\right)\right)=\Delta_{7}^{\prime}\left(\iota_{7}\right) .
$$

Since $\rho: S^{3} \rightarrow R P^{3}=S_{3}$ is a double covering projection, the induced map $B \rho: H P^{\infty}$ $=B S^{3} \rightarrow B S O_{3}$ is a fibration with its fiber $B Z_{2}=K\left(Z_{2}, 1\right)$. Then, if $n>2$, we have the composite of isomorphisms

$$
\pi_{n}\left(H P^{\infty}\right)=\pi_{n}\left(B S^{3}\right) \stackrel{B \rho_{*}}{\cong} \pi_{n}\left(B S O_{3}\right) \stackrel{a d}{\cong} \pi_{n-1}\left(S O_{3}\right),
$$

where $a d$ denotes the adjoint isomorphism. Since $\pi_{3}\left(S_{3}\right)=Z\{\rho\}$, there exists a map $\rho^{\prime} \in \pi_{4}\left(H P^{\infty}\right)$ such that,

$$
a d \circ B \rho_{*}\left(\rho^{\prime}\right)=\rho \text { and } \pi_{4}\left(H P^{\infty}\right)=Z\left\{\rho^{\prime}\right\} .
$$

Furthermore, according to the celluar approximation theorem, we have

$$
\rho^{\prime}=j_{2} \circ j_{1},
$$

where the maps $j_{1}: S^{4} \rightarrow H P^{2}=S^{4} \bigcup_{\nu_{4}} e^{8}$ and $j_{2}: H P^{2} \rightarrow H P^{\infty}$ denote the natural inclusion maps. Let $c\left(\xi_{m}\right) \in \pi_{4}\left(B S O_{3}\right)$ and $c\left(\nu_{4}^{*} \xi_{m}\right) \in \pi_{7}\left(B S O_{3}\right)$ denote the characteristic classes of the $S^{2}$-bundles $\xi_{m}$ and $\nu_{4}^{*} \xi_{m}$. We put $\rho^{\prime \prime}=B \rho_{*}\left(\rho^{\prime}\right) \in \pi_{4}\left(B S O_{3}\right)$. Since $\chi\left(X_{m}\right)=m \rho$, according to (5.8) and (5.9), we have

$$
c\left(\boldsymbol{\xi}_{m}\right)=m\left(B \boldsymbol{\rho}_{*}\left(\boldsymbol{\rho}^{\prime}\right)\right)=m \boldsymbol{\rho}^{\prime \prime} .
$$

Since $\left[\iota_{4}, \iota_{4}\right]=2 \nu_{4} \pm E \omega$ and $H\left(\nu_{4}\right)=\iota_{7}$,

$$
\begin{aligned}
c\left(\boldsymbol{\nu}_{4}^{*} \xi_{m}\right) & =\boldsymbol{\nu}_{4}^{*} c\left(\xi_{m}\right) \\
& =c\left(\xi_{m}\right) \circ \nu_{4} \\
& =\left(m \rho^{\prime \prime}\right) \circ \nu_{4} \\
& =m\left(\rho^{\prime \prime} \circ \nu_{4}\right)+(m(m-1) / 2)\left[\rho^{\prime \prime}, \boldsymbol{\rho}^{\prime \prime}\right] \circ H\left(\iota_{4}\right) \\
& =m\left(\boldsymbol{\rho}^{\prime \prime} \circ \nu_{4}\right)+(m(m-1) / 2) \boldsymbol{\rho}^{\prime \prime} \circ\left[\iota_{4}, \iota_{4}\right] \circ \iota_{7} \\
& =m\left(\boldsymbol{\rho}^{\prime \prime} \circ \nu_{4}\right)+(m(m-1) / 2) \boldsymbol{\rho}^{\prime \prime} \circ\left(2 \nu_{4} \pm E \boldsymbol{\omega}\right) \\
& =m^{2}\left(\boldsymbol{\rho}^{\prime \prime} \circ \nu_{4}\right) \pm(m(m-1) / 2)\left(\boldsymbol{\rho}^{\prime \prime} \circ E \boldsymbol{\omega}\right) .
\end{aligned}
$$

Because $H P^{2}$ is a mapping cone of $\nu_{4}, j_{1} \circ \nu_{4}=0$. Hence $\rho^{\prime \prime} \circ \nu_{4}=\left(B \rho \circ \rho^{\prime}\right) \circ \nu_{4}=$ $B \rho \circ\left(j_{2} \circ j_{1}\right) \nu_{4}=0$, and

$$
c\left(\nu_{4}^{*} \xi_{m}\right)= \pm(m(m-1) / 2)\left(\rho^{\prime \prime} \circ E \boldsymbol{\omega}\right)= \pm(m(m-1) / 2) B \rho_{*}\left(\rho^{\prime}\right) \cdot E \boldsymbol{\omega} .
$$

Thus, according to (5.8) and (5.9), we obtain

$$
\chi(X)= \pm(m(m-1) / 2) \rho \circ \omega,
$$

where $\chi(X) \in \pi_{6}\left(B S O_{3}\right)$ denotes the characteristic element of $\nu_{4}^{*} \xi_{m}$. Then, if $p: S O_{3} \rightarrow S O_{3} / S O_{2}=S^{2}$ is a natural projection map, $p_{*}(\rho)=\eta_{2}$ and 


$$
\begin{aligned}
\Delta_{7}\left(\nu_{4}\right) & =\Delta_{7}^{\prime}\left(\iota_{7}\right) \\
& =p_{*}(\chi(X)) \\
& = \pm(m(m-1) / 2) p_{*}(\rho) \circ \omega \\
& = \pm(m(m-1) / 2)\left(\eta_{2} \circ \omega\right) .
\end{aligned}
$$

This completes the proof.

Q.E.D.

Proof of Proposition 5.3. Consider the homotopy exact sequence (5.4). First, we suppose $m \equiv 1(\bmod 2)$. Then according to (5.6), we have

$$
\pi_{6}\left(X_{m}\right)=Z_{(12, m)}\left\{(j \circ i)_{*}\left(\eta_{2} \circ \omega\right)\right\}=Z_{(m, 3)}\left\{(j \circ i)_{*}\left(\eta_{2} \circ \omega\right)\right\} \cong \begin{cases}0 & \text { if }(m, 3)=1 \\ Z_{3} & \text { if }(m, 3)=3 .\end{cases}
$$

Now, we assume that $m=2 m^{\prime} \neq 0$ for some integer $m^{\prime}$. According to (5.6), the following is exact:

$$
0 \longrightarrow Z_{\left(12, m^{\prime}\right)}\left\{\eta_{2} \circ \omega\right\} \stackrel{(j \circ i)_{*}}{\longrightarrow} \pi_{6}\left(X_{m}\right) \stackrel{p_{m *}}{\longrightarrow} \pi_{6}\left(S^{4}\right) \longrightarrow 0 .
$$

Since $\left(m \eta_{2}\right) \circ \eta_{3}^{2}=m\left(\eta_{2}^{3}\right)=0$, there exists a coextension of $\eta_{3}^{2}, \tilde{\eta} \in \pi_{6}\left(L_{m}\right)$ such that,

$$
q_{m} \circ \tilde{\eta}=\eta_{4}^{2} .
$$

Furthermore, because $\pi_{6}\left(L_{m}\right)$ is a finite group, the order of $\tilde{\eta}$ is 2 . Hence it follows from $p_{m} \mid L_{m}=q_{m}$ that the sequence (5.13) is split exact. Hence $\pi_{6}\left(X_{m}\right)$ $=Z_{\left(12, m^{\prime}\right)}\left\{(j \circ i)_{*}\left(\eta_{2} \circ \omega\right)\right\} \oplus Z_{2}\{\tilde{\eta}\} \cong Z_{\left(12, m^{\prime}\right)} \oplus Z_{2}$ Q. E. D.

Proof of Theorem 1.1.

The assertion easily follows from (4.8), (5.2) and (5.3). $\quad$ Q. E. D.

Proof of Proposition 1.3.

Let $m$ be an integer which satisfies the condition $(m, 6)=3$. Then it follows from the proof of (5.3) that $\pi_{6}\left(X_{m}\right)=Z_{3}\left\{(j \circ i)_{*}\left(\eta_{2} \circ \omega\right)\right\}$. According to [19], $\pi_{6}\left(S^{3}\right)$ $=Z_{12}\{\omega\}=Z_{4}\left\{\nu^{\prime}\right\} \oplus Z_{3}\left\{\alpha_{1}(3)\right\}$. Hence $(j \circ i)_{*}\left(\eta_{2} \circ \omega\right)=(j \circ i)_{*}\left(\eta_{2} \circ \alpha_{1}(3)\right)$. Thus, it follows from (1.1) that the group $E q\left(X_{m}\right)$ is generated by two elements,

$$
\theta_{1}=\lambda\left((j \circ i)_{*}\left(\eta_{2} \circ \alpha_{1}(3)\right)\right) \text { and } \theta_{2}^{\prime},
$$

where $\theta_{2}$ denotes the map in $\left[X_{m}, X_{m}\right]$ which satisfies the conditions,

$$
\phi\left(\theta_{2}\right)=h_{1} \in E q\left(L_{m}\right)=Z_{2}\left\{h_{1}\right\} \quad \text { and } \quad \phi\left(\theta_{2}\right)=1 \in E q\left(S^{4}\right)=Z_{2}=\{ \pm 1\} .
$$

According to (3.3), the suspension homomorphism $\Sigma: E q\left(L_{m}\right) \rightarrow E q\left(\Sigma L_{m}\right)$ is monomorphic. Therefore

$$
\Sigma \theta_{2} \neq i d_{\Sigma X_{m}} \text { and }\left(\Sigma \theta_{2}\right) \circ\left(\Sigma \theta_{2}\right)=\imath d_{\Sigma X_{m}} .
$$


Since $E\left(\eta_{2} \circ \alpha_{1}(3)\right)=\eta_{3} \circ E \alpha_{1}(3)=0$, according to the naturality of the Barcus-Barratt operation $\lambda$, we have

$$
\begin{aligned}
\Sigma \theta_{1} & =\Sigma \lambda\left((\jmath \circ i)_{*}\left(\eta_{2} \circ \alpha_{1}(3)\right)\right) \\
& =\lambda\left(\Sigma(j \circ i)_{*}\left(E\left(\eta_{2} \circ \alpha_{1}(3)\right)\right)\right) \\
& =\lambda(0) \\
& =\imath d_{\Sigma x_{m}} .
\end{aligned}
$$

Then it follows from (5.15) and (5.17) that we obtain

$$
\operatorname{Im}\left[\Sigma: E q\left(X_{m}\right) \longrightarrow E q\left(\Sigma X_{m}\right)\right]=Z_{2}\left\{\Sigma \theta_{2}\right\} \cong Z_{2} .
$$

This completes the proof.

Q.E. D

\section{REFERENCES}

[1] W.D, Barcus AND M.G. Barratt, On the homotopy classiflcation of a fixed map, Tran. Amer. Math. Soc. 88 (1958), 57-74.

[2] I. M. JAmEs, On the homotopy groups of certain pairs and triads, Quart. J. Math. Oxford, 5 (1954), 260-270.

[3] I. M. James, Note on cup-products, Proc. Amer. Math. Soc., 8 (1957), 374-383.

[4] I. M. James, On sphere bundles over spheres, Comment. Math. Helv., 35 (1961), $126-135$.

[5] I. M. James AND J.H.C. Whitehead, The homotopy theory of sphere bundles over spheres (I), Proc. London Math. Soc., 4 (1954), 196-218.

[6] I. M. James and J.H.C. Whitehead, The homotopy theory of sphere bundles over spheres (II), Proc. London Math. Soc., 5 (1955), 148-166.

[7] P.J. KAHN, Self-equivalences of $(n-1)$-connected $2 n$-manifolds, Math. Ann., 180 (1969), 26-47.

[8] J. Levine, Self-equivalences of $S^{n} \times S^{k}$, Trans. Amer. Math. Soc., 143 (1969), 523-543.

[9] M. Mimura and N. Sawashita, On the group of self-homotopy equivaleces of principal $S^{3}$-bundles over spheres, Hiroshima Math. J., 14 (1984), 415-424.

[10] Y. Nomura, Self-homotoy equivalences of Stiefel manifolds $W_{n, 2}$ and $V_{n, 2}$, Osaka J. Math., 20 (1983) , 79-93.

[11] S. ОкA, Groups of self-equivalences of certain complexes, Hiroshima Math, J., 2 (1972), 285-293.

[12] S. OKa, N. Sawashita and M. Sugawara, On the group of self-equivalences of a mapping cone, Hiroshima Math. J., 4 (1974), 9-28.

[13] I. W. RUTTER, The group of self-homotopy equivalences of principal three sphere bundles over the seven sphere, Math. Proc. Camb. Phil. Soc., 84 (1978), 303-311.

[14] J.W. Rutter, The group of homotopy self-equivalence classes of $C W$ complexes, Math. Proc. Camb. Phil. Soc., 93 (1983), 275-293.

[15] S. SASAO, On self-homotopy equivalences of the total spaces of a sphere bundle over a sphere, Kodai Math. J., 7 (1984), 365-381.

[16] S. SASAO, On self-homotopy equivalences of $S^{3}$-principal bundles over $S^{n}$, Kodai Math. J., 8 (1985), 285-295. 
[17] N. SaWAshita, On the group of self-equivalences of the product of spheres, Hiroshima Math. J., 5 (1975), 69-86.

[18] A. J. Sieradski, Twisted self-homotopy equivalences, Pacific J. Math., 34 (1970), 789-802.

[19] H. TodA, Composition methods in homotopy groups of spheres, Annals of Math. Studies, 49 (1962), Princeton Univ. Press.

[20] K. Yamaguchi, On the homotopy type of $C W$ complexes with the form $S^{2} \cup e^{4} \cup e^{6}$, Kodai Math. J., 5 (1982), 303-312.

[21] K. Yamaguchi, Corrections on the homotopy type of $C W$ complexes with the form $S^{2} \cup e^{4} \cup e^{6}$, Kodai Math. J., 6 (1983), 443.

[22] K. YAmAgUCHI, The group of self-homotopy equivalences of $S^{2}$-bundles over $S^{4}$. II Applications, preprint.

Department of Mathematics,

Tokyo Institute of Technology,

Oh-OKayama, Meguro, Tokyo, Japan. 